Chimia 46(1992) 477-479

C Neue Schweizerische Chemische Gesellschaft ISSN 0009-4293

\section{TXRF Spektrometer zum Nachweis von Spurenelementen}

Ferenc Hegedüi*

Abstract. The TXRF (total reflection X-ray fluorescence) spectrometer is a very useful instrument for measuring ultratrace elements in aqueous solutions, in metals, in minerals, in biological and environmental samples etc. Elements with atomic number $>13$ are detected simultaneously with a minimum detection limit of pg. The sample preparation is very simple and only few $\mu \mathrm{g}$ or $\mu \mathrm{l}$ sample material is required.

\section{Einleitung}

In den letzen Jahren hat sich die Anwendung des TXRF (Total Reflection XRay Fluorescence) Spektrometer rasch verbreitet. Der Grund dafür ist, dass dieses Spektrometer in vielen Probenarten sehr kleine, pg-Mengen von Spurenelementen relativ einfach und schnell nachweisen kann. Laut Veröffentlichungen an der PIXCAM Konferenz 1991 [1], wurde die TXRF Methode für Spurenelement-Analyse auf folgenden Gebieten verwendet:

- Süsswasser: Regen- und Grundwasser, Abfluss von Kläranlagen, Flüsse, Leitungswasser etc.

- Schwermetalle in Bodenproben (z.B $\mathrm{Pb}, \mathrm{Hg}, \mathrm{Cd}$ )

- Biologie (z.B. Gewebe von Pflanzen und Tieren)

- Medizin (z.B. $\mathrm{Zn}$ in Haut oder Se in Blut)

- Luftverschmutzung: Staub- und Aerosol-Proben

- Geologie und Mineralogie

- Oberflächenkontamination von Halbleiterkristallen

Für eine Analyse wird nur sehr wenig Probenmaterial benötigt. Deshalb sind einige Probleme nur mit dieser Methode lösbar. Ein Beispiel aus der Biologie ist der Nachweis von $\mathrm{Hg}$ in der einige MilliGramm schweren Lebewesens.

Besonders gut geeignet ist die Methode für Wasseranalysen. Die Messprobe ist sehr schnell vorbereitet durch Pipettiegramm schweren Leber eines einige rung von $5 \mu$ auf die Quarzglasplatte und Trocknung im Vakuum.

Am Paul Scherrer Institut wurden mit Hilfe des TXRF-Spektrometers ganz spezielle Probleme gelöst [2-4]. Ein solches Problem ergab sich in einem nuklearen Reaktor-Sicherheitsexperiment. Iodhaltiges Gas wurde durch eine einige Meter hohe Wassersäule geblasen. Die Konzentrationsabnahme des Iods wurde mit TXRF gemessen, viel einfacher und billiger als mit der Radioiod-Methode. Ein anderes spezielles Problem war, in reinen Metallen die Konzentration der Transmutationselemente zu messen. Wird ein Element mit Hochenergie-Protonen oder -Neutronen bombardiert, werden Transmutationen erzeugt, z.B. Ni, $\mathrm{Co}, \mathrm{Fe}$ in $\mathrm{Cu}$. Alle diese Konzentrationen sind hier sehr niedrig und das Matrix-Element stört die Messungen. Es wurde mono-energetische Synchroton-Strahlung statt Röntgen-Röhren verwendet. Auf diese Weise konnte der KNM-Wert unter 1 pg gebracht werden.

\section{Beschreibung des Spektrometers}

Fig. 1 und 2 zeigen das Arbeitsprinzip des Spektrometers [5]. (In Fig. 1 und 2 ist ein grosser, $\mathrm{ca} .40^{\circ}$, Winkel eingezeichnet, damit die Abbildung übersichtlich wird.) Eine sehr dünne Schicht der zu untersuchenden Probe liegt auf einer Quarzplatte. Ein fein kollimierter Röntgen-Strahl trifft auf diese Oberfläche unter einem sehr kleinen (einige min) Winkel. Dieser primäre Röntgen-Strahl regt die Proben-Atome so an, dass diese ihre charakteristische Röntgen-Strahlung emittieren. Das Spektrum dieser Strahlung wird mit Hilfe eines über der Probe liegenden $\mathrm{Si}(\mathrm{Li})$ Detektors gemessen. Fig. 3 zeigt ein gemessenes Spektrum mit den charakteristischen Röntgen-Linien der Atome des Probenmaterials.

Die Anregung des Atoms erfolgt auf folgende Weise: Ein Photon des primären Strahls übergibt seine Energie an ein Elektron. Wenn diese genügend gross ist, wird das Elektron aus seiner Bahn herausgestossen. An seine Stelle wird ein Elektron von einer äusseren Schale springen. Die Energiedifferenz zwischen den zwei Schalen wird von einem Photon weggetragen, das man charakteristisches Röntgen-Photon nennt. Da die Bindungsenergie der Elektronschale eine Funktion der Ordnungszahl ist, ist das Atom anhand der Energie der des emittierten Photons identifizierbar. Nehmen wir das Fe-Atom als Beispiel. Die Bindungsenergie des Elektrons in der innersten $\mathrm{K}$ Schale ist 7.11 $\mathrm{keV}$. Wird dieses herausgestossen, springt an seine Stelle ein Elektron von der LIII Schale (Bindungsenergie: $0.71 \mathrm{keV}$ ) und es entsteht eine für Fe charakteristische Ka Röntgen-Linie mit einer Energie von $6.40 \mathrm{keV}$. Gleichzeitig können auch andere Röntgen-Photonen entstehen, weil an die leere Stelle der LIII Schale ein Elektron von den äusseren Schalen (N oder M) springen wird. In diesem Fall wird die

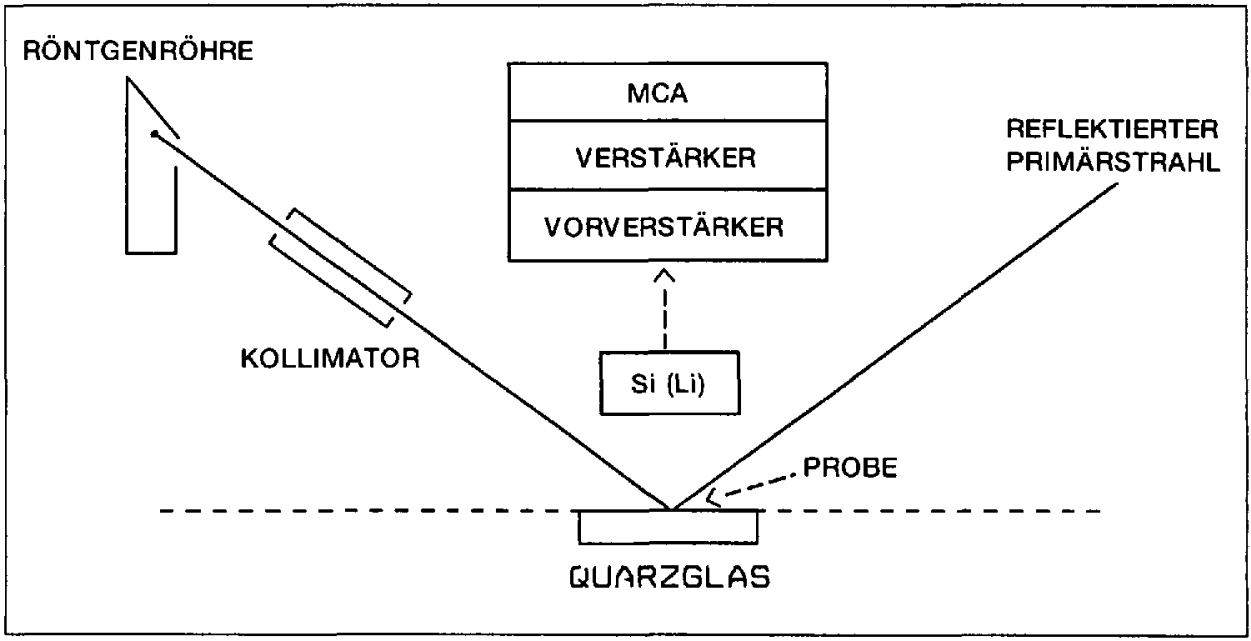

Fig. I. Prinzip des TXRF-Spektrometers 
emittierte Photonenenergie viel kleiner, nämlich $<0.8 \mathrm{keV}$ sein. $\mathrm{Da}$ der $\mathrm{Si}(\mathrm{Li})$ Detektor nur ber $1.3 \mathrm{keV}$ wirksam ist, sind nur die charakteristischen Röntgen-Linien der Schalen K und L detektierbar. Man sollte bemerken, dass bei den Elementen mit tieferer Ordnungszahl ( $\mathrm{Z}<13)$ (Rönt gen-Energie $<1.3 \mathrm{keV}$ ) die Möglichkeit besteht einen $\mathrm{Si}(\mathrm{Li})$-oder HPGe-Detektor mit Diamantfenster zu verwenden. Die untere Energieschwelle liegt dann bei nur $0.5 \mathrm{keV}$.

Da der Eintrittswinkel des primären Strahls kleiner ist als der kritische Winkel, dringt der Strahl (bis auf eine Penetrationstiefe von 20-50 nm) nicht ins Quarzglass ein, sondern wird total-reflektiert, d.h. praktisch ohne Streuung und ohne Anregung der Atome (Fig. 2c). Deswegen ist der Untergrund, bzw. die Nachweisgrenze, extrem niedrig, das ist der grosse Vorteil des TXRF gegenüber den normalen XRF Spektrometer-Typen.

\section{Einstellung des Spektrometers}

Die Auswahl des primären RöntgenStrahls hängt von der Zusammensetzung der zu analysierenden Probe ab. Der Anregungwirkungsquerschnitt ist dann gross, wenn die Energie des Primärstrahls nicht viel grösser ist als die Energie der anzuregenden Elektronenschale. Zum Beispiel: will man Co nachweisen, ist es das beste, eine Röntgen-Röhre mit Cu-Anode zu verwenden, weil im Primärstrahl die $\mathrm{Cu}-\mathrm{Ka}$ (8.05 keV) Linie dominiert und gerade oberhalb der Energie der Co-K-Schale (7.71 keV) liegt. Will man in einer Probe gleichzeitig mehrere Elemente nachweisen, sollte man einen Primärstrahl wählen, dessen Energie-Spektrum für alle analysierenden Elemente akzeptabel ist. In der Praxis werden Röntgen-Röhren mit folgenden Anoden benützt: $\mathrm{Cr}(\mathrm{Ka}: 5.41 \mathrm{keV})$, $\mathrm{Cu}(\mathrm{Ka}: 8.05 \mathrm{keV}), \mathrm{Mo}(\mathrm{Ka}: 17.4 \mathrm{keV})$ und W(La: 8.4, Lb: $9.7 \mathrm{keV}$ ). Zusätzlich zur charakteristischen Röntgen-Strahlung des Anodenmaterials, emittiert die RöntgenRöhre auch Bremsstrahlung. Dieses Spektrum hängt von der Hochspannung ab. In manchen Fällen könnte man die Bremsstrahlung nutzen, die $\mathrm{K}$-Schalen höherer Z-Elemente anzuregen. In anderen Fällen, dei denen nur kleinere Energien benötigt werden, ist die Bremsstrahlung unerwünscht, weil sie den Untergrund erhöht. Wird nach dem Kollimator ein Spiegel eingesetzt und dessen Winkel so eingestellt, dass nur der kleinere Energie-Anteil des Spektrums total reflektiert wird, kann die Bremsstrahlung mit Streuung weitgehend eliminiert werden.

Bei jeder Umstellung des Spektrometers soll der optimale Eintrittswinkel am Probenträger-Quarzplatte eingestellt werden. Der kritische Winkel steigt mit abnehmender Energie des Primärstrahls. In der Praxis liegt der Eintrittswinkel im Bereich zwischen 2-10 min. Eine so feine

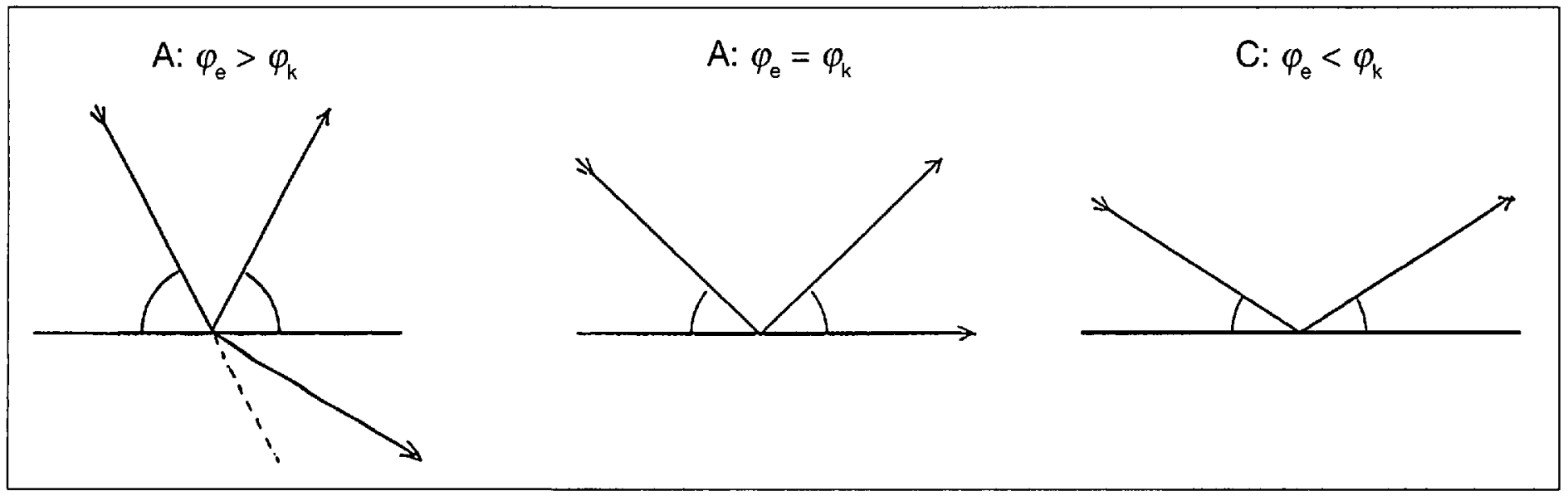

Fig. 2. Eintritt, Brechung und Reflektion des Röntgen-Strahls auf Quarzglas. $\varphi_{\mathrm{e}}$ : Eintrittswinkel; $\varphi_{\mathrm{k}}$ : kritischer Winkel.

Fig. 3. Röntgen-Spektrum einer Multielement-Standardprobe (S: $100 \mathrm{ng}, \mathrm{K}: 100 \mathrm{ng}, \mathrm{Ti}: 60 \mathrm{ng}, \mathrm{Mn}$ : $100 \mathrm{ng}, \mathrm{Fe}: 68 \mathrm{ng}, \mathrm{Co}: 100 \mathrm{ng}, \mathrm{Ni}$ : $100 \mathrm{ng}$ )

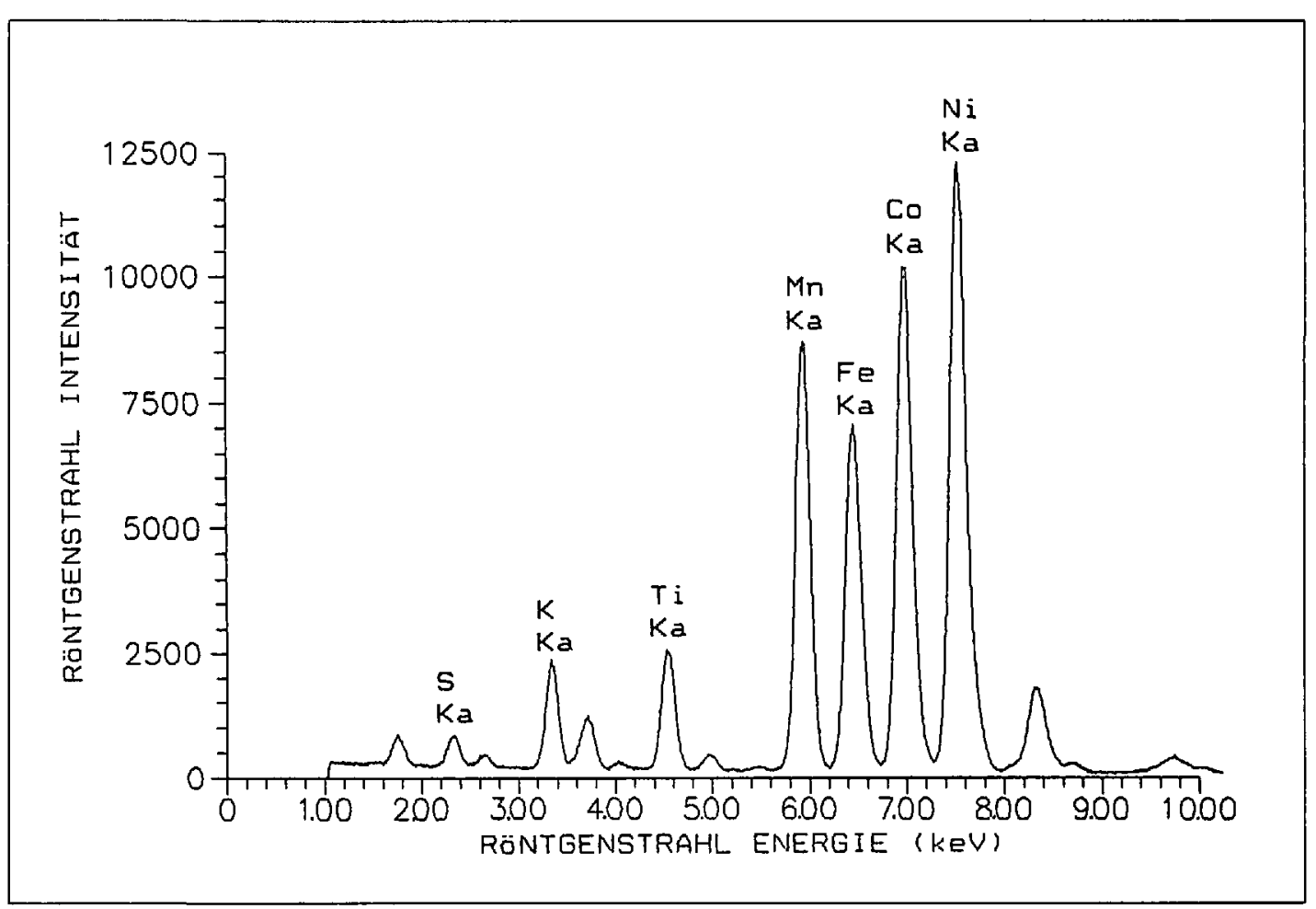




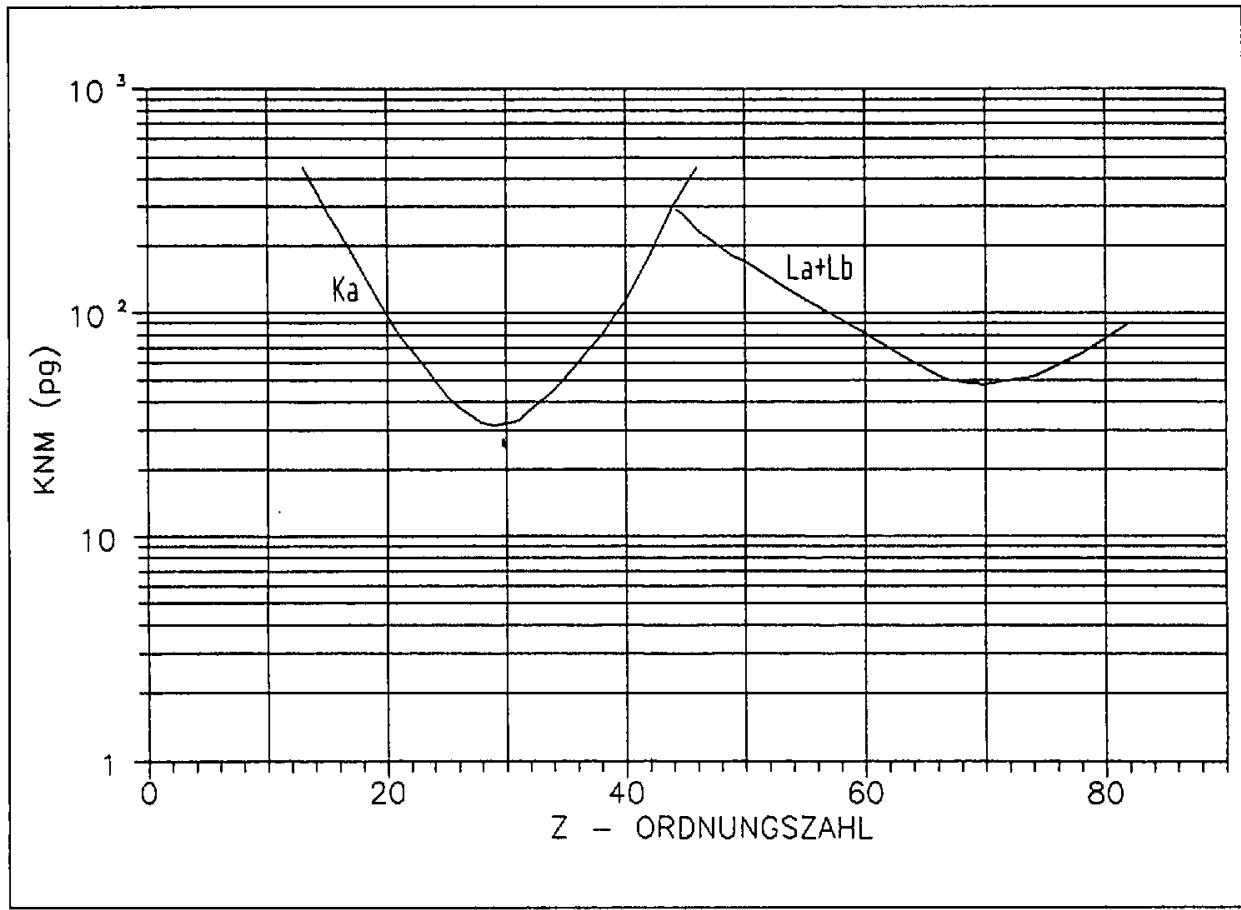

Fig. 4. Kleinste nachweisbare Menge in Funktion der Ordnungszahl, W-Röhre



Fig. 5. Kleinste nachweisbare Menge in Funktion der Ordnungszahl, Mo-Röhre

Einstellung ist nur mit einer sehr präzisen und reproduzierbaren feinmechanischen Einrichtung möglich. Die runde Quarzplatte wird gegen drei Stahlkugeln gedrückt. Auf diese Weise ist seine Position sehr genau definiert. Die Einstellung erfolgt durch die Bewegung der Stahlkugeln. Die Reproduzierbarkeit der Position der Stahlkugeln ist $<1 \mu$ (entspricht etwa $0.1 \mathrm{~min}$ ).

Nach jeder Umstellung sollte das Spektrometer mit Hilfe von Standardproben kalibriert werden. Fig. 3 zeigt ein solches Kalibrier-Spektrum mit der folgenden Einstellung: Röntgenröhre Anodenmaterial: W; Hochspannung: $55 \mathrm{keV}$; Strahl Kolli- zuweisen. Die Grösse der 'kleinsten nachweisbaren Menge' (KNM) hängt hauptsächlich von der Zusammensetzung des Probenmaterials und von der Einstellung des Spektrometers ab. Zum Beispiel liegt in Regen- und Boden-Wasserproben der KNM-Wert (im Bereich: $13<\mathrm{Z}<92$ ) zwischen 5-500 pg. Enthält die Probe Matrixelemente, z.B. Salz in Meerwasser, wird der KNM-Wert höher. Um den Untergrund tief zu halten, soll die zu untersuchende Probenmenge $<5 \mu \mathrm{g}$ (oder für dünne Schichten: $50 \mathrm{~nm}$ ) sein. Fig. 4 zeigt die Änderung des KNM-Wertes in Funktion der Ordnungszahl, wobei eine WolframRöhre als Primärstrahl-Quelle verwendet wurde. Der Vorteil der Wolfram-Röhre ist, dass gleichzeitig praktisch alle Elemente messbar sind; deren Nachteil, dass der KNM Wert relativ hoch, z.B. bei Blei $90 \mathrm{pg}$, ist. Bei einer anderen Einstellung mit Mo-Röhre, wird dieser Wert $<5$ pg sein (s. Fig. 5).

Eingegangen am 18. September 1992

[1] Pacific-International Congress on X-Ray Analytical Methods (PICXCAM), Proceedings, Adv. X-Ray Anal. 1992, 35.

[2] F. Hegedüs, P. Winkler, 'Low Level lodine Detection by TXRF Spectrometry', Adv. $X$ Ray Anal. 1989, 32, 251.

[3] F. Hegedüs, P. Wobrauschek, W.F. Sommer, R.W. Ryon, Ch. Streli, P. Winkler, P. Ferguson, P. Kregsamer, R. Rieder, M. Victoria, A. Horsewell, 'Total Reflection X-Ray Fluorescence Spectrometry of Metal Samples Using Synchrotron Radiation at SSRL', Proceedings of the European EDXRS Workshop, 1992, to be published in X-Ray Spectrometry.

[4] F. Hegedüs, P. Winkler 'Uranium Concentration Measurement in Water Samples with TXRF', Adv. X-Ray Anal. 1992, 35.

[5] F. Hegedüs, P. Winkler, P. Wobrauschek, Ch. Streli, 'TXRF Spektrometer for Trace Element Detection', Adv. X-Ray Anal. 1990, $33,5 \mathrm{Bl}$. mation: $5 \times 0.05 \mathrm{~mm}$; Eintrittswinkel: 5 min. Da im Spektrum des Primärstahls die Wolfram $\mathrm{La}(8.4 \mathrm{keV})$ und $\mathrm{Lb}(9.7 \mathrm{keV})$ Linien dominieren, ist die Intensität der $\mathrm{Ni}$ K-Linie am stärksten. Die Intensität der anderen Elemente nimmt mit abnehmender Ordnungszahl ab.

\section{Kleinste nachweisbare Menge}

Das Spektrometer ist sehr geeignet einerseits Spurenelemente in kleinen Probenmengen zu messen, anderseits Oberflächen-Verunreinigungen an flachen Sioder anderen Halbleiter-Kristallen nach- 